\title{
Acupuncture for acute low back pain: a systematic review and meta-analysis
}

\author{
Xuan Su ${ }^{1}$, Hong Qian ${ }^{1}$, Biyu Chen ${ }^{1}$, Wenjuan Fan ${ }^{2}$, Danghan $\mathrm{Xu}^{3} \wedge$, Chunzhi Tang ${ }^{2}$, Liming Lu ${ }^{4}$ \\ ${ }^{1}$ Guangdong Provincial Hospital of Traditional Chinese Medicine Integrated with Western Medicine, Guangzhou University of Chinese Medicine, \\ Guangzhou, China; ${ }^{2}$ Medical College of Acu-Moxi and Rehabilitation, Guangzhou University of Chinese Medicine, Guangzhou, China; ${ }^{3}$ The \\ First Affiliated Hospital of Guangzhou University of Chinese Medicine, Guangzhou, China; ${ }^{4}$ Clinical Research and Data Center, South China \\ Research Center for Acupuncture and Moxibustion, Medical College of Acu-Moxi and Rehabilitation, Guangzhou University of Chinese Medicine, \\ Guangzhou, China \\ Contributions: (I) Conception and design: X Su, H Qian; (II) Administrative support: L Lu, C Tang; (III) Provision of study materials or patients: X \\ Su, H Qian; (IV) Collection and assembly of data: B Chen, W Fan; (V) Data analysis and interpretation: B Chen, D Xu; (VI) Manuscript writing: All \\ authors; (VII) Final approval of manuscript: All authors. \\ Correspondence to: Liming Lu. Clinical Research and Data Center, South China Research Center for Acupuncture and Moxibustion, Medical College \\ of Acu-Moxi and Rehabilitation, Guangzhou University of Chinese Medicine, Guangzhou 510006, China. Email: lulimingleon@126.com.
}

Background: Acupuncture has been widely used for acute low back pain (LBP), yet there remains continued controversy regarding its efficacy. Therefore, we aimed to critically evaluate the evidence for acupuncture as an effective treatment for acute LBP.

Methods: English and Chinese databases were searched for randomized controlled trials (RCTs) that involved acupuncture for acute LBP published up to May 2020. Data on the outcomes of pain intensity, functional status, and analgesic use were extracted. The meta-analysis was performed using the Cochrane Collaboration's RevMan 5.3, and pooled data were expressed as mean differences (MD) with 95\% confidence intervals (CIs).

Results: Of the 13 eligible RCTs identified, 11 RCTs (involving 707 patients) provided moderate-quality evidence that acupuncture has a statistically significant association with improvements in VAS (visual analog scale) score [MD: -1.75 (95\% CI: -2.39, -1.12)]. Two studies indicated that acupuncture did not influence the RMDQ (Roland-Morris Disability Questionnaire) scores more than the control treatment [MD: -2.34 (95\% CI: $-5.34,0.67)$ ]. Three studies suggested that acupuncture influenced the ODI (Oswestry Disability Index) scores more than the control treatment [MD: -12.84 (95\% CI: -23.94, -1.74)]. Two studies suggested that acupuncture influenced the number of pills more than the control treatment [MD: -3.19 (95\% CI: $-3.45,-2.92)]$.

Conclusions: Acupuncture treatment of acute LBP was associated with modest improvements in the VAS score, ODI score, and the number of pills, but not the RMDQ score. Our findings should be considered with caution due to the low power original studies. High-quality trials are needed to assess further the role of acupuncture in the treatment of acute LBP.

Keywords: Acupuncture; acute low back pain; randomized controlled trial; systematic review; meta-analysis

Submitted Oct 10, 2021. Accepted for publication Jan 22, 2021.

doi: 10.21037/apm-20-1998

View this article at: http://dx.doi.org/10.21037/apm-20-1998

^ ORCID: 0000-0001-9491-5815. 


\section{Introduction}

Low back pain (LBP) is the leading cause of disability worldwide. In 2015, the point prevalence of limitedmobility LBP reached $7.3 \%$ globally, which means that up to 540 million people suffered from LBP at any one time (1). Acute LBP is normally self-limiting, with $90 \%$ of cases experiencing significant relief from pain and disability within 1 month (2,3). However, recurrences of symptoms are very common and lead to serious medical and economic problems for individuals and society (4).

Muscle relaxants, non-steroidal anti-inflammatory drugs (NSAIDs), and opioids are the most commonly used medications to treat acute LBP in emergency departments across the United States (5). Due to the adverse effects associated with drug treatments, such as gastrointestinal symptoms and cardiovascular events (6,7), an increasing number of patients are choosing to combine medications with complementary and alternative medical (CAM) treatments to reduce pain (8).

Acupuncture is a safe CAM therapy that is frequently used to treat pain $(9,10)$. A 2002 study in the United States found that back pain was the primary reason patients visited acupuncturists. Nevertheless, several previous systematic reviews of acupuncture for acute LBP have yielded inconsistent results (11-13), and the most recent meta-analysis included randomized controlled trials (RCTs) published up to 2013, most of which had methodological issues and were unreliable. Considering that several highquality studies have since been published, we conducted a systematic review and meta-analysis to critically evaluate the evidence for the efficacy of acupuncture to treat acute LBP.

We present the following article in accordance with the PRISMA reporting checklist (available at http://dx.doi. org/10.21037/apm-20-1998).

\section{Methods}

\section{Search strategy}

The following databases were searched from their inception until May 2020: the Allied and Complementary Medicine Database (AMED), the Cochrane Central Register of Controlled Trials, Embase, Medline, as well as Chinese databases including the China National Knowledge Infrastructure database (CNKI), the Wanfang database, and the Chinese Science and Technology Periodicals database (VIP). Medical Subject Heading (MeSH) terms, words appearing in the title or abstract, and keywords were used in the search strategy (details are shown in Appendix 1).

\section{Study selection}

\section{Types of studies}

All published RCTs concerning acupuncture for treating acute LBP, limited to Chinese and English language reports, were included. Conference papers, systematic reviews, case reports, research protocols, and experimental animal studies were excluded. Studies with incomplete data were also excluded.

\section{Types of participants}

Participants aged $\geq 18$ years that suffered from acute LBP for $\leq 12$ weeks, of which symptoms were discomfort and pain positioned below the costal margin and above the subgluteal fold, regardless of primary or secondary and with or without associated leg pain, were included. Pregnant women and patients with critical illnesses were excluded.

\section{Types of interventions}

The experimental group interventions were acupuncture therapy, including manual acupuncture, electroacupuncture, and auricular acupuncture. Studies on acupoint injection, acupoint embedding, massage, moxibustion, cupping, and other therapies and studies on acupuncture combined with other therapies were not included.

\section{Types of controls}

The controls could be sham acupuncture, analgesic, or blank controls; however, trials in which the control was acupuncture or traditional Chinese medicine interventions were excluded.

\section{Types of outcomes}

This meta-analysis's primary outcome was the visual analog scale (VAS) (used for pain assessment; scores from 0 to 10, with higher scores indicating a higher degree of pain). Secondary outcomes were the Roland-Morris Disability Questionnaire (RMDQ) (used for lumbar mobility function assessment; scores from 0 to 24, with higher scores representing more severe lumbar dysfunction), the Oswestry Disability Index (ODI) (used for assessment of pain level and daily self-care ability; scores from 0 to 100 , with higher scores, signifying more severe lumbar dysfunction), and numbers of pills (NOP) (with larger doses indicating a higher degree of pain and deeper analgesic drug dependence). Studies with no related outcomes were excluded. 


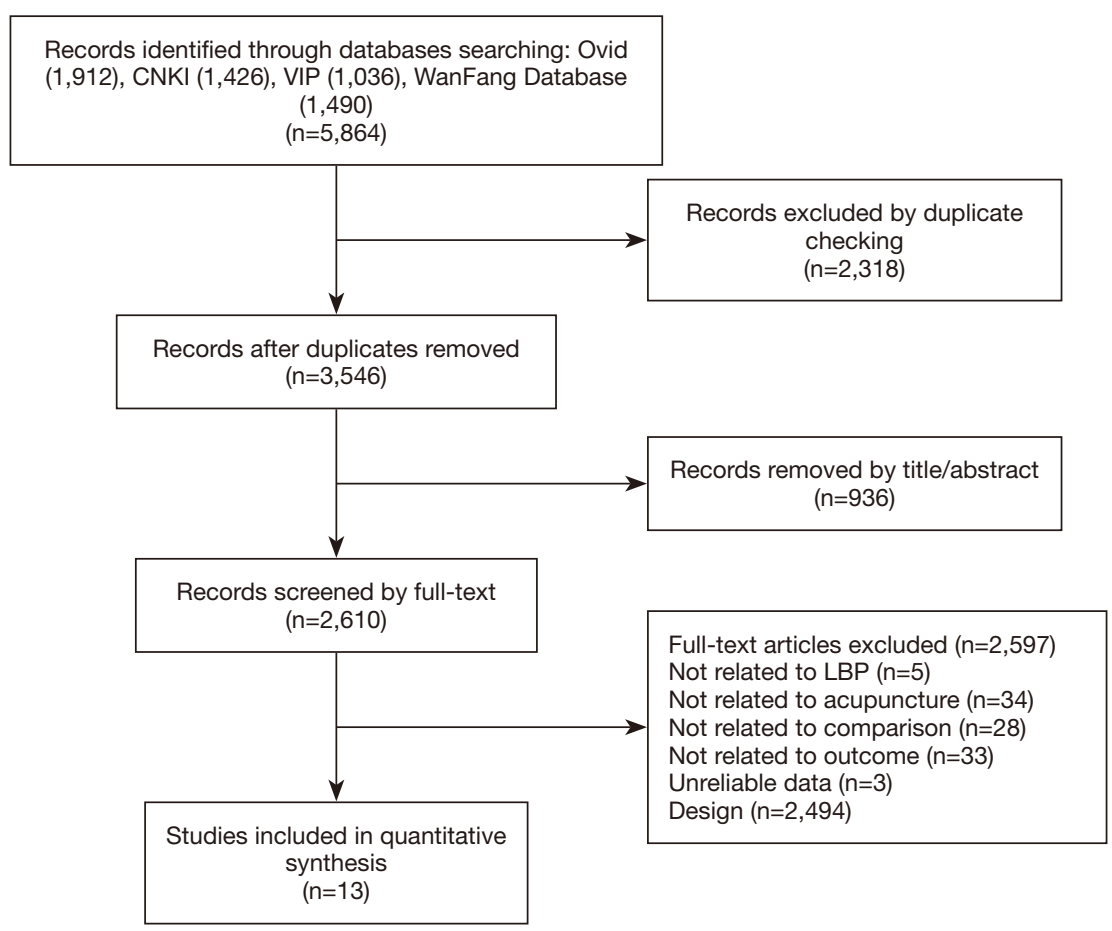

Figure 1 Article selection process.

\section{Data extraction and risk of bias assessment}

Two reviewers (Su Xuan and Qian Hong) independently screened the literature. Papers that did not satisfy the inclusion criteria were eliminated. The full texts of potentially eligible studies were obtained and further analyzed. The required information, including the topic, year of publication, authors, number of participants, ages, samples, interventions, controls, and outcomes, were extracted and organized into a data extraction table. Two reviewers (Su Xuan and Qian Hong) independently assessed the eligible papers' risk of bias using the Cochrane tool (14). The consistency evaluation of risk-of-bias assessment was examined using the kappa coefficient. A third reviewer (Biyu Chen) resolved differences through discussion.

\section{Data synthesis and analysis}

This review was conducted using Review Manager software, version 5.3(Copenhagen, Denmark: The Nordic Cochrane Center, The Cochrane Collaboration, 2013). Dichotomous variables were assessed through relative risk (RR). The mean difference (MD) was used for continuous variables, and the $95 \%$ confidence interval (CI) was calculated. A $\mathrm{P}$ value $<0.05$ was considered statistically significant. If heterogeneity was not significant $\left(\mathrm{I}^{2} \leq 50 \%\right.$ or $\left.\mathrm{P} \geq 0.10\right)$, a fixed-effects model was adopted to analyze the pooled effects. A random-effects model was employed when heterogeneity was statistically significant $\left(\mathrm{I}^{2}>50 \%\right.$ or $\mathrm{P}<0.10)$. We also performed subgroup analysis in terms of the type of control, course of treatment, and acupoint selection to examine the sources of heterogeneity and determine whether the above conditions would affect our conclusions. We also carried out sensitivity analysis from different study qualities to further confirm our conclusions. Moreover, we assessed the risk of publication bias.

\section{Results}

\section{Study characteristics}

A total of 5,864 articles were obtained from the electronic databases, and 2,318 duplicate publications were excluded; after preliminary exclusion of 936 papers by reading the titles and abstracts, the remaining 2,610 papers needed to be further filtered by reading the full texts. Finally, 13 papers satisfied the inclusion criteria (15-27). The literature screening process is presented in Figure 1.

A total of 13 RCTs involving 899 participants were included in the review. There were 461 cases in the acupuncture group 
and 438 cases in the matched group. There were nine studies from China (19-27) and one each from Brazil (15), the United Kingdom (16), Australia (17), and South Korea (18). Four studies were published in English (15-18), and nine were published in Chinese (19-27). The participants' ages ranged from 18 to 75 years. In the intervention group, the main therapeutic methods were manual acupuncture $(16-18,21,24,25)$, electroacupuncture $(22,23,27)$, beryllium acupuncture (26), wrist and ankle acupuncture (20), floating acupuncture (19), and Yamamoto's acupuncture (15).

Four studies used acupuncture combined with lower back exercises $(18,21,24,25)$. Seven studies required de qi for acupuncture manipulation, a specific sensation such as soreness, numbness, or swelling (16,21-25,27). One study did not allow any needle sensation or reaction (20), and five studies did not specify whether or not needle sensation was achieved $(15,17-19,26)$. Five studies used a fixed acupuncture point selection scheme $(15,18,20,21,27)$, and eight studies used personalized acupoints according to the patients' conditions $(16,17,19,22-26)$. Three studies compared acupuncture with sham needling, which means that the needle only touches the skin at the same point but does not penetrate it $(15,16,20)$. Ten studies compared acupuncture with Western medicine (17-19,21-27). Eleven studies that included 707 participants reported pain intensity on a $100-m m$ VAS $(15,16,19-27)$. Four studies assessed lumbar spine function through the ODI (17-19,24), and two studies used the RMDQ to evaluate lumbar function $(15,16)$. Two studies assessed pain intensity according to the number of painkillers the patients took $(15,16)$. Table 1 presents the principal characteristics of the included studies.

\section{Quality assessment}

Among the 13 included reports, five were rated as high quality $(15-18,20)$ and eight as low quality $(19,21-27)$. Among all of the studies, 11 mentioned specific methods for random sequence generation, of which six used computers to generate the random sequence $(15-20)$ and five used the random number table method (21-24,26); two studies did not mention the process of random sequence generation $(25,27)$. Three studies described the methods for allocation concealment in detail $(16,18,20)$, while the other studies did not indicate whether allocation concealment was employed (15,17,19,21-27). Among the 13 studies, three described blinding of the participants and the outcome assessment $(15,16,20)$, two mentioned blinding of the outcome assessment only $(17,18)$, and the others did not report a blinding method (19,21-27). There were 10 studies with no cases lost to follow-up $(15,19-27)$, two studies that used intention-to-treat analysis to deal with cases that were lost to follow-up $(16,18)$, and one study that did not address the treatment of data for cases that were lost to follow-up (17). In all of the included studies, we did not find selective reporting or other sources of bias. Items 2, 3, and 5 gained substantial agreement between the two assessors, and items 1, 4, 6, and 7 acquired perfect consistency. The risk of bias summary and its consistency evaluation are displayed in Figures 2 and 3 and Table S1.

\section{Meta-analysis}

\section{Primary outcome: VAS}

Eleven studies $(15,16,19-27)$ involving 707 patients used the VAS to evaluate acupuncture's effectiveness. Since there was high heterogeneity, a random-effects model was adopted. The pooled results indicated a greater improvement in VAS scores in the acupuncture group compared to the control group [MD: $\left.-1.75(-2.39,-1.12), \mathrm{P}<0.00, \mathrm{I}^{2}=93 \%\right]$ (Figure 4).

\section{Subgroup analysis}

(I) For different comparators

(i) Acupuncture vs. sham acupuncture

Three trials $(15,16,20)$ compared acupuncture with sham acupuncture and evaluated the effectiveness of acupuncture by VAS. The analysis revealed that acupuncture did not have a greater effect on the VAS score than sham acupuncture [MD: $\left.-0.99(-1.24,-0.73), \mathrm{P}=0.63, \mathrm{I}^{2}=0 \%\right]$ (Figure 5).

(ii) Acupuncture vs. drugs

Eight trials (19,21-27) compared acupuncture with drugs and evaluated the effectiveness of acupuncture by VAS. The analysis showed that acupuncture had a greater effect on the VAS score than drugs [MD: $-2.00(-2.83,-1.17), \mathrm{P}<0.00$, $\left.\mathrm{I}^{2}=93 \%\right]$ (Figure 5).

The subgroup differences test indicated that different comparators might lead to heterogeneity $(\mathrm{P}=0.02)$ (Figure 5).

(II) For different durations of intervention

(i) 1 day or less

In two trials $(20,25)$, the patients were treated once for 1 day or less, and the effectiveness of acupuncture was evaluated using the VAS. The analysis indicated a greater effect on the VAS score in the acupuncture group than in the control group [MD: $-1.60(-2.56$, -0.64), $\mathrm{P}=0.001, \mathrm{I}^{2}=80 \%$ ] (Figure 6). 


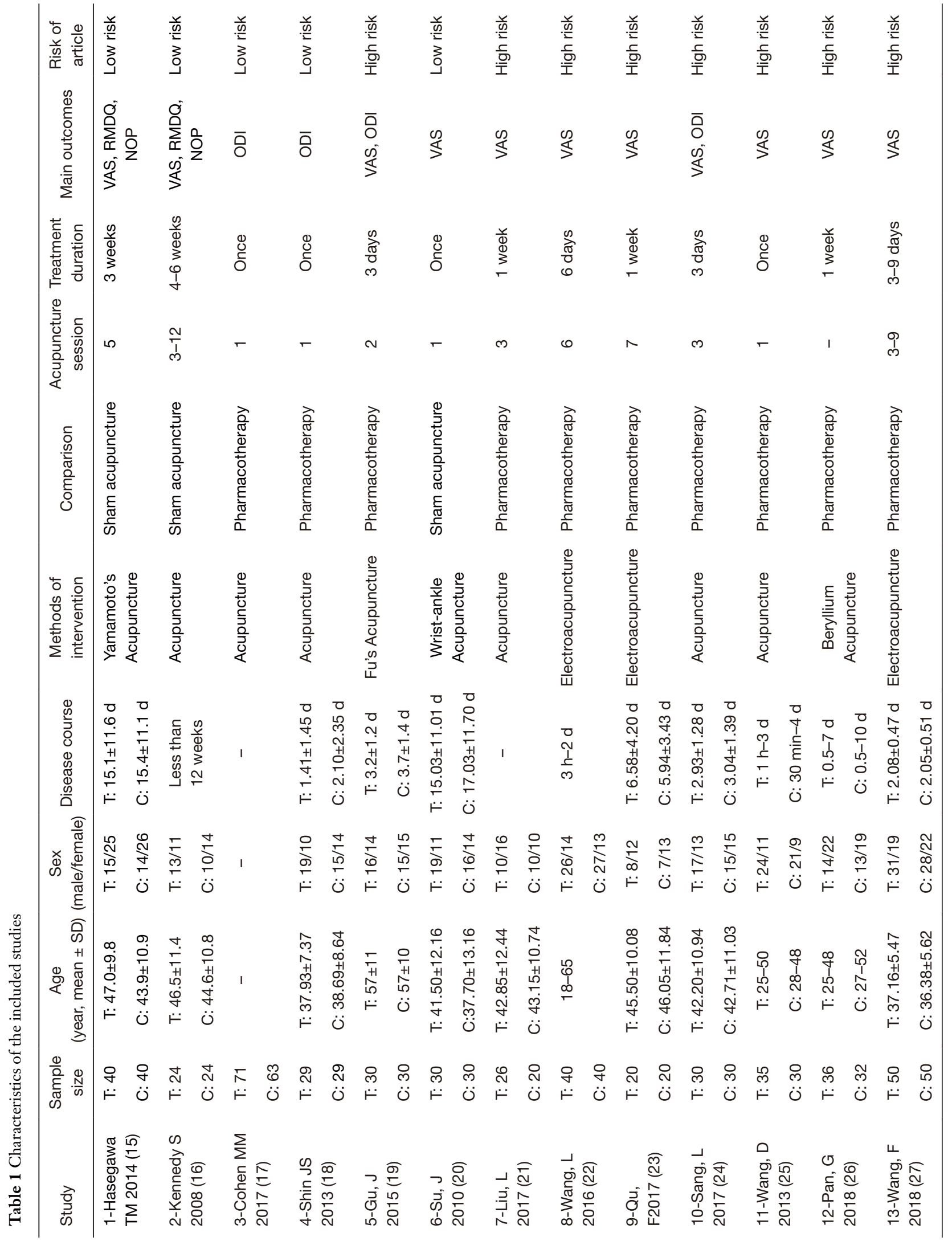




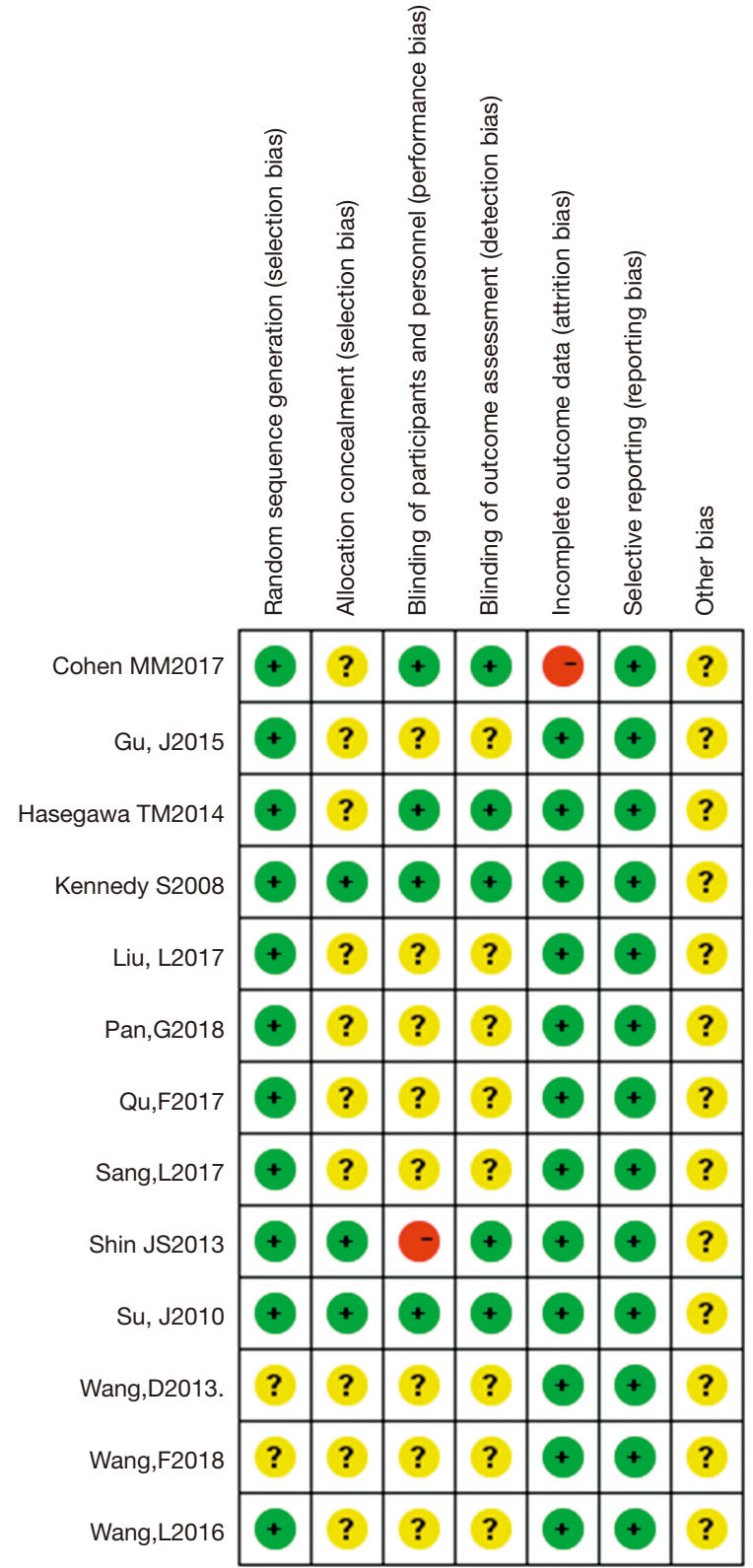

Figure 2 Article selection process.

\section{(ii) Between 1 day and 2 weeks}

In seven trials $(19,21-24,26,27)$, the patients were treated for between 1 day and 2 weeks. The frequency of treatment in one study (21) was once for 2 days; in another (19) it was twice for 3 days; and in the remaining studies (22-24,26,27), it was once daily. The effectiveness of acupuncture was evaluated by the VAS. The analysis indicated a greater effect on the VAS score in the acupuncture group than in the control group [MD: $-1.98(-2.92$, -1.04 ), $\mathrm{P}<0.00, \mathrm{I}^{2}=94 \%$ ] (Figure 6).

(iii) 2 weeks or more

In two trials $(15,16)$, patients were treated for 2 weeks or more, and the frequencies of treatment were both once or twice a week. The VAS evaluated the effectiveness of acupuncture. The analysis indicated a greater effect on the VAS score in the acupuncture group than in the control group [MD: $\left.-0.93(-1.23,-0.63), \mathrm{P}<0.00, \mathrm{I}^{2}=0 \%\right]$ (Figure 6).

The test of subgroup differences indicated that the different treatment durations might not lead to heterogeneity $(\mathrm{P}=0.06)$ (Figure 6).

(III) For different acupoints selection

(i) Local acupoints selection

In six trials $(16,19,22,23,26,27)$, local acupoints were selected for treatment, and the VAS evaluated the effectiveness of acupuncture. The analysis indicated a greater effect on the VAS score in the acupuncture group than in the control group [MD: $-1.54(-2.51$, -0.57 ), $\mathrm{P}<0.00, \mathrm{I}^{2}=95 \%$ ] (Figure 7).

(ii) Distal acupoints selection

In six trials $(15,20,21,24,25)$, patients in the acupuncture group were treated with distal acupoints selection, and the analysis showed that acupuncture had a greater effect on the VAS score than the control group [MD: $-2.02(-2.76,-1.29)$, $\mathrm{P}<0.00, \mathrm{I}^{2}=83 \%$ ] (Figure 7).

The subgroup differences test indicated that different acupoint selection might not lead to heterogeneity $(\mathrm{P}=0.44)$ (Figure 7).

\section{Sensitivity analysis}

(I) Low-risk

Analysis of the three trials $(15,16,20)$ that were evaluated as low-risk indicated a greater effect on the VAS score in the acupuncture group than in the control group [MD: $-0.99(-1.24,-0.73), \mathrm{P}<0.00$, $\left.\mathrm{I}^{2}=0 \%\right]$ (Figure 8).

(II) Unclear/high-risk

The analysis of the eight trials $(19,21-27)$ that were evaluated as unclear or high-risk indicated a greater effect on the VAS score in the acupuncture group than in the control group [MD: $-2.00(-2.83,-1.17)$, $\mathrm{P}<0.00, \mathrm{I}^{2}=93 \%$ ] (Figure 8).

The subgroup differences test indicated that different trial risks might lead to heterogeneity $(\mathrm{P}=0.02)$ (Figure 8). However, the result in the low-risk group indicated that there was no statistical heterogeneity, 


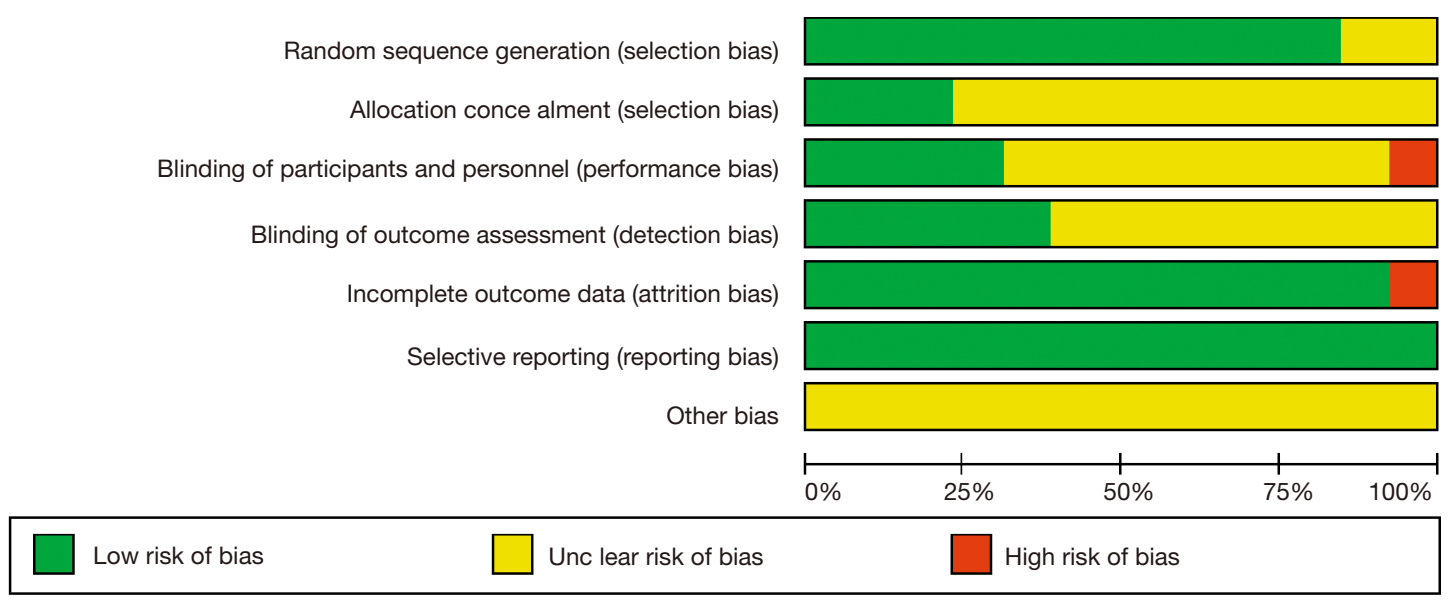

Figure 3 Risk of bias chart.

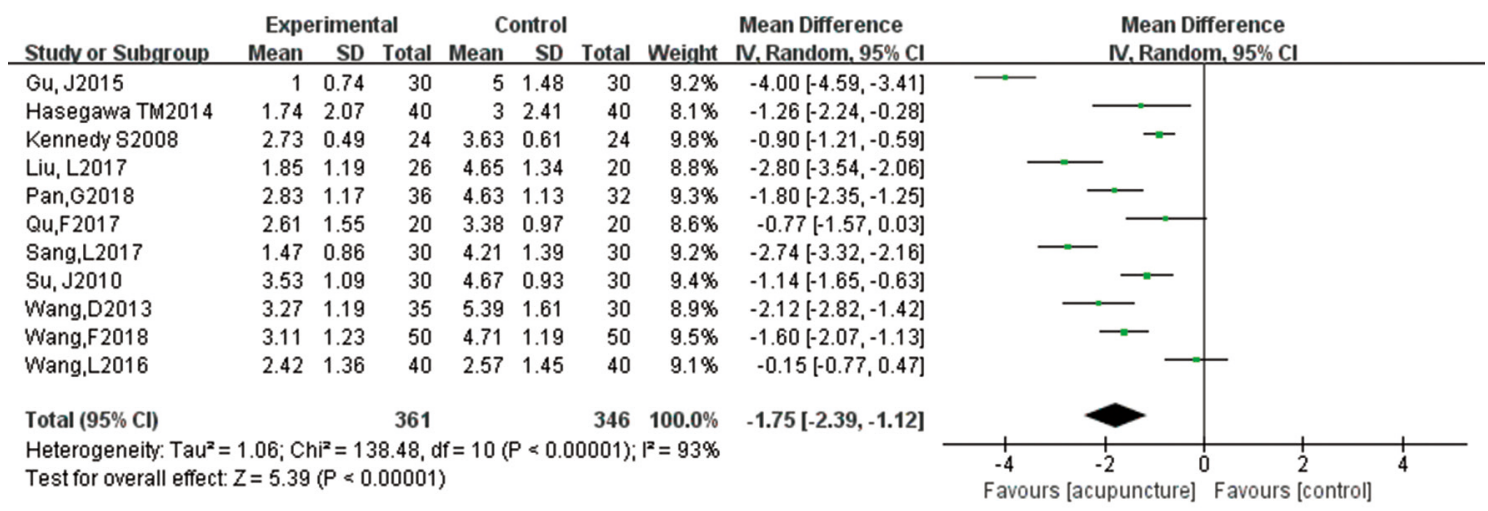

Figure 4 The forest plot of the acupuncture $v s$. control groups for VAS score.

whereas the result in the unclear/high-risk group indicated that there was statistical heterogeneity. This suggested that the heterogeneity may result from the trial risk.

(III) Publication bias

The funnel plot (Figure 9) for assessing the risk of publication bias appeared to be relatively symmetrical, suggesting that low publication bias existed in this meta-analysis.

\section{Secondary outcomes \\ $R M D Q$}

Two studies $(15,16)$ used the RMDQ to evaluate the effectiveness of acupuncture. The analysis indicated that acupuncture did not influence the RMDQ scores more than sham acupuncture $[\mathrm{MD}:-2.34(-5.34,0.67), \mathrm{P}=0.13$, $\mathrm{I}^{2}=84 \%$ ] (Figure 10).

\section{$O D I$}

Three studies $(17,19,24)$ used the ODI to evaluate the effectiveness of acupuncture. The analysis indicated that acupuncture influenced the ODI scores more than medication [MD: -12.84 (-23.94, -1.74), $\mathrm{P}=0.02, \mathrm{I}^{2}=96 \%$ ] (Figure 11).

\section{NOP}

Two studies $(15,16)$ used NOP to evaluate the effectiveness of acupuncture. The analysis indicated that acupuncture influenced the NOP more than sham acupuncture [MD: -3.19 (-3.45, -2.92), $\left.\mathrm{P}<0.00001, \mathrm{I}^{2}=0 \%\right]$ (Figure 12).

\section{Safety}

One high-quality study reported no side effects in either the acupuncture or the sham group (15), three trials $(17,19,20)$ mentioned that there were mild side effects, and the remaining trials did not report side effects. Therefore, we believe that acupuncture was safe in this analysis. 


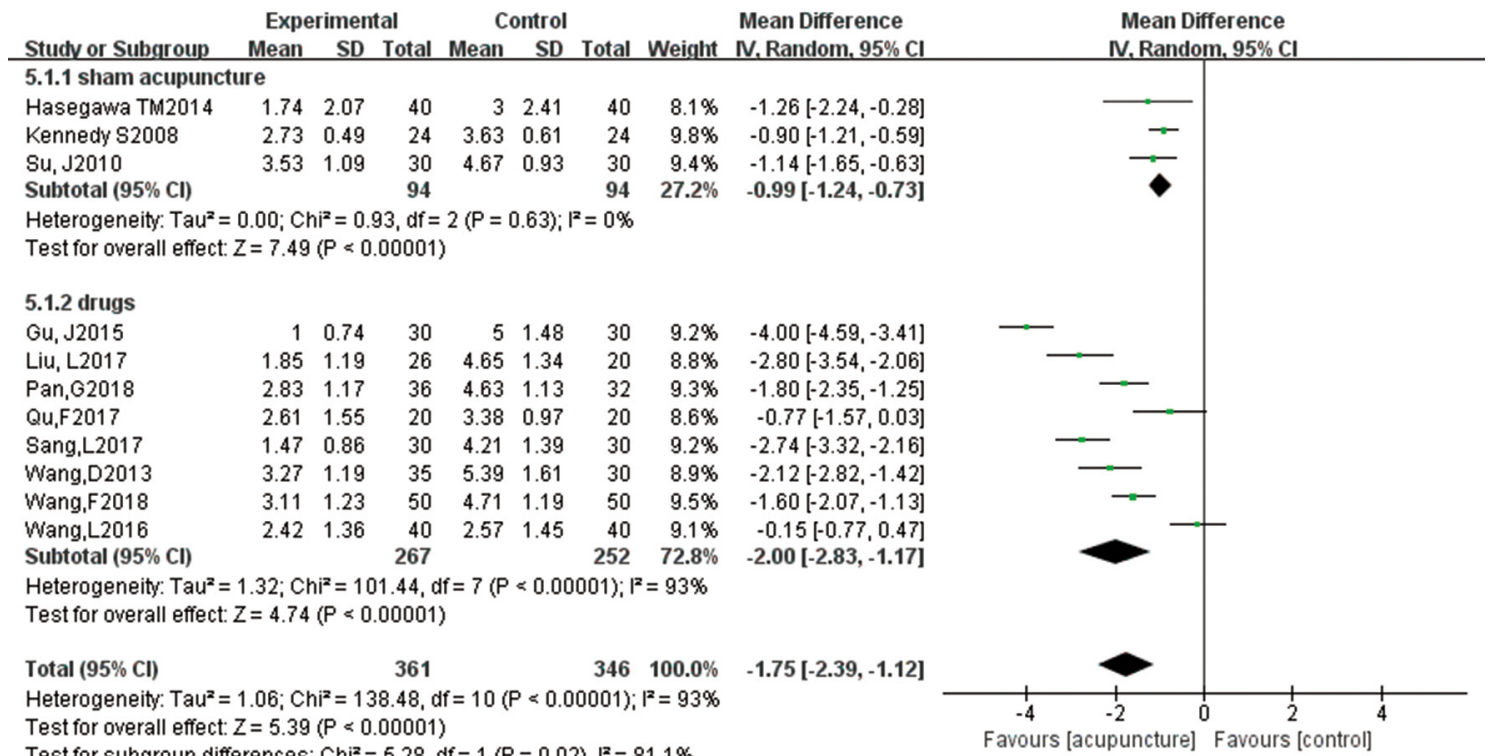

Figure 5 The forest plot of acupuncture $v s$. sham acupuncture/drugs for VAS score.

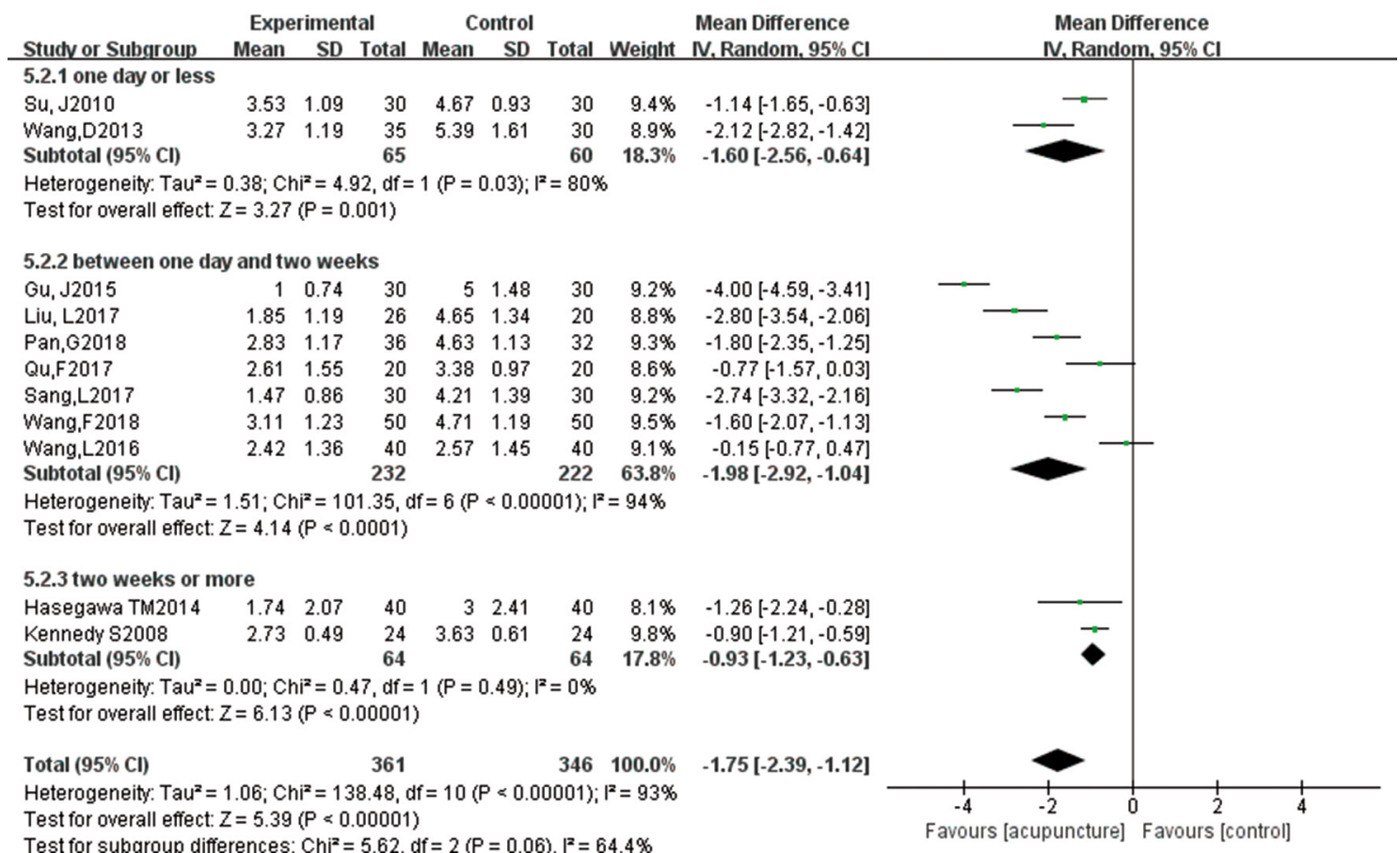

Figure 6 The forest plot of different treatment durations for VAS score.

\section{Discussion}

\section{Summary of findings}

This meta-analysis included 13 RCTs comparing acupuncture with drugs or sham acupuncture to treat patients with acute LBP. The included RCTs involving a total of 899 participants. This analysis's main conclusion was that acupuncture treatment for acute LBP showed 


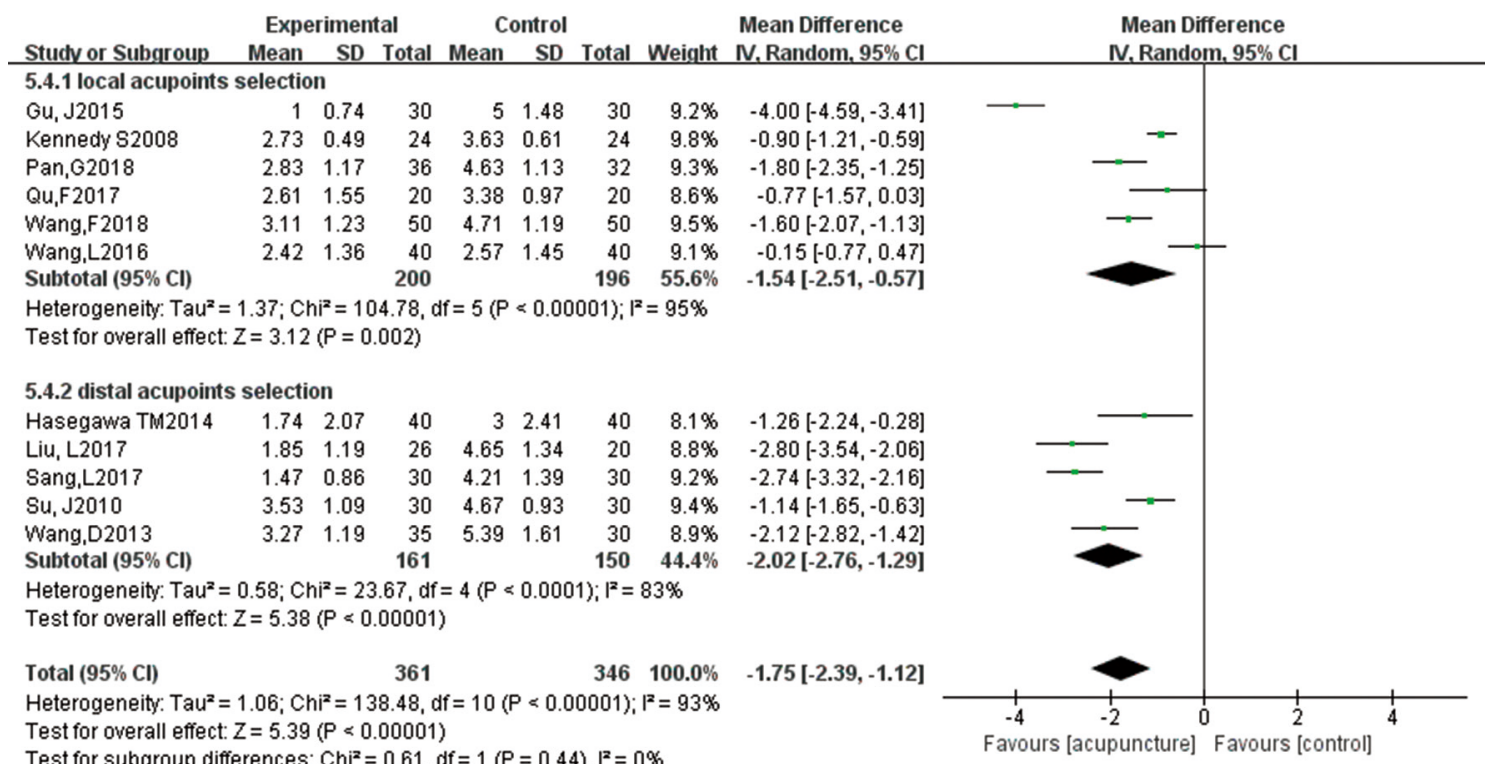

Figure 7 The forest plot of different acupoints selection for VAS score.

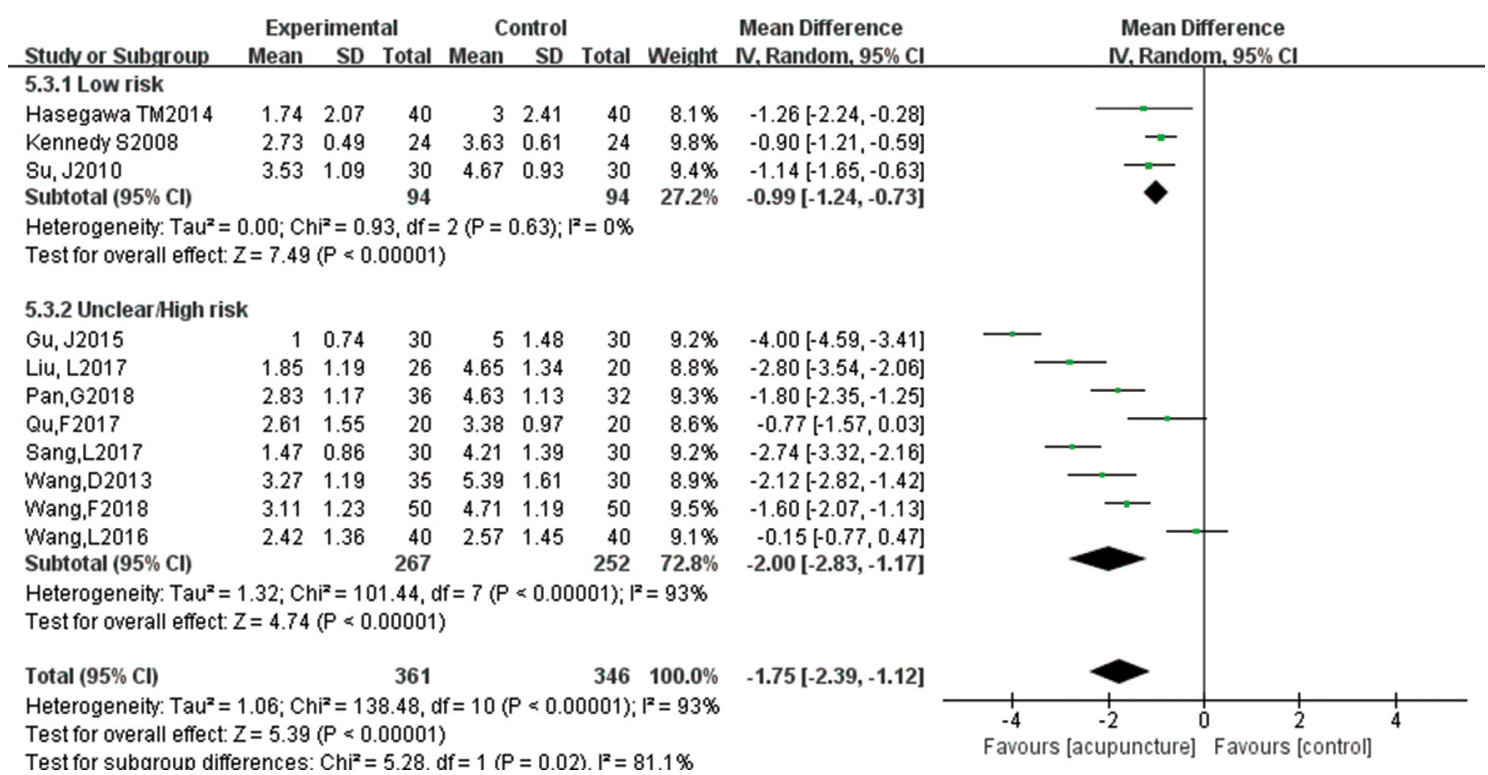

Figure 8 The forest plot of different article risks and VAS.

statistically significant benefits in VAS score, ODI score, and NOP. However, the effect of acupuncture on the RMDQ scores was not greater than that of the control group. The heterogeneity was high for the VAS results, and the subgroup analysis suggested that this may be because of different comparators and trial risks, but could not be explained by different treatment durations.

\section{Findings concerning previous reports}

In a previous meta-analysis published in 2020, researchers found that acupuncture could reduce the pain in (sub)acute and chronic non-specific LBP when the VAS and RMDQ scores were compared with sham or placebo acupuncture used as outcomes (28). In our paper, we focused only on acute LBP and selected the VAS, ODI, and RMDQ scores 
and the NOP as outcomes, which reflected the effectiveness of acupuncture therapy on more levels. In a meta-analysis and systematic review published in 2015, researchers found that acupressure, acupuncture, and cupping could effectively

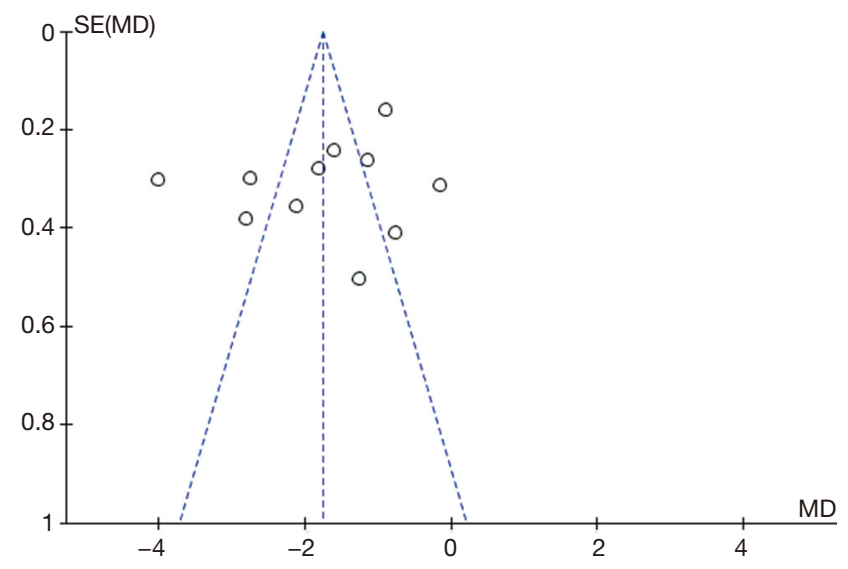

Figure 9 Funnel plot of publication bias. treat the disability and pain associated with chronic LBP (29). Our analysis focused on acute LBP, selected acupuncture treatment as the intervention, and medication or sham acupuncture as control groups, and found similar results.

At present, the outcomes of studies on the treatment of LBP were mainly symptom scales. The VAS was used for pain assessment, and the ODI and RMDQ both reflected dysfunction in patients with acute LBP. However, the ODI was less sensitive in assessing LBP with mild and moderate dysfunction, whereas the RMDQ was the opposite. The ODI assessed patients' pain and dysfunction with acute LBP, whereas the RMDQ only assessed the dysfunction caused by acute LBP and lacked an assessment of pain severity $(30,31)$. In this paper, acupuncture therapy of acute LBP was associated with modest improvements in the ODI score but not in the RMDQ score. This was probably because in treating mild and moderate acute LBP, acupuncture treatment did not show its comparative advantage, or because acupuncture alleviated pain but caused the same improvement in lumbar mobility

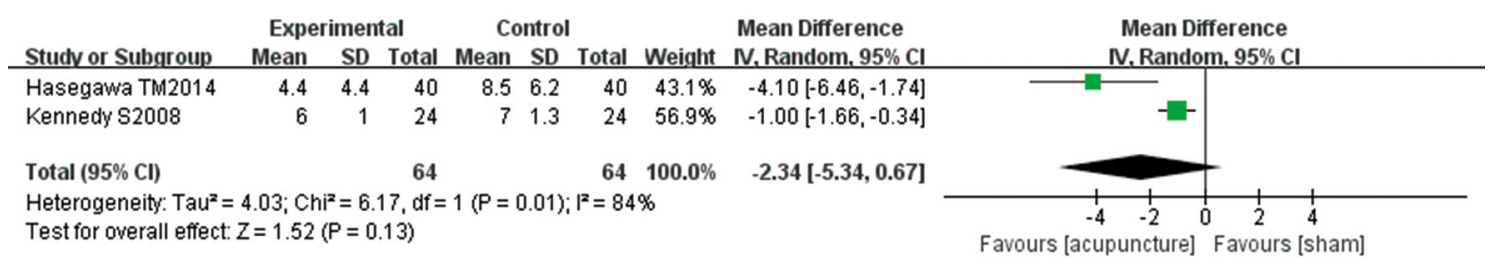

Figure 10 The forest plot of acupuncture $v s$. sham acupuncture for RMDQ.

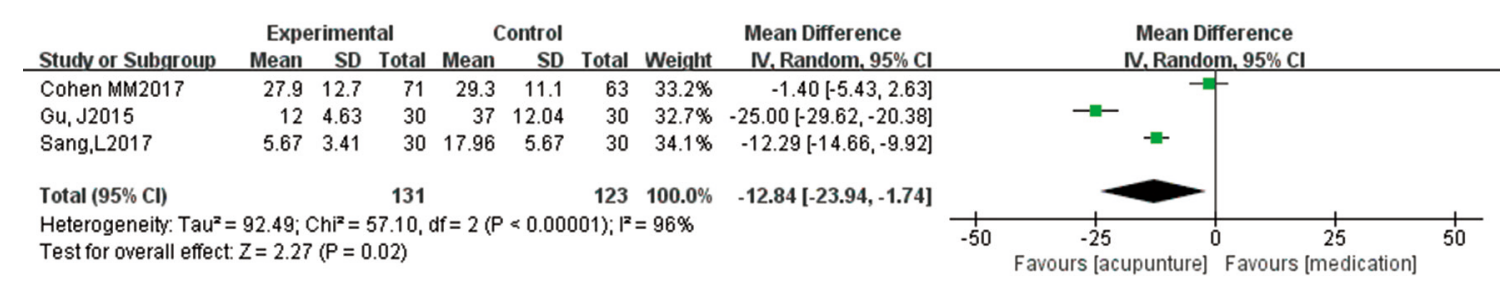

Figure 11 The forest plot of acupuncture $v s$. medication for ODI.

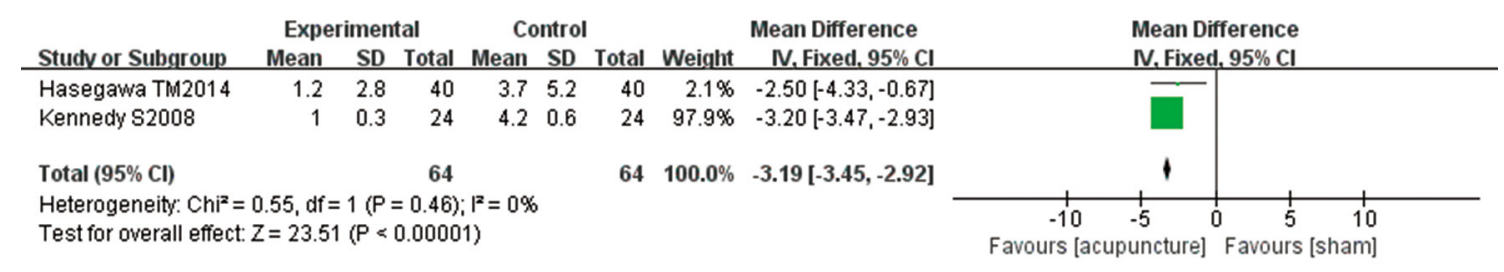

Figure 12 The forest plot of acupuncture $v s$. sham acupuncture for NOP. 
as the control treatment. NOP directly reflected the effect of acupuncture therapy in reducing dependence on analgesics.

All in all, both our study and previous studies have demonstrated that acupuncture has significant effects in alleviating acute LBP symptoms on different levels, and it is obvious that acupuncture could be used to treat acute LBP.

\section{Superiority}

Firstly, our search for literature related to acupuncture treatment of acute LBP encompassed the four foreign databases and all relevant Chinese literature (through the three major databases in China).

Furthermore, acupuncture therapy has a long history and is more widely used in China compared to other countries. Therefore, there were numerous studies about acupuncture treatment of acute LBP. The inclusion of Chinese literature enabled us to incorporate more literature from a wider range and gain a deeper understanding of the specific operation methods and actual efficacy of acupuncture in the treatment of acute LBP.

Our study's major strength was that we chose the VAS, ODI, RMDQ, and NOP as outcomes, which reflected the effectiveness of acupuncture therapy on more levels.

\section{Limitations}

Firstly, limitations in the quantity and quality of the original research were difficult to ignore. There were only 13 trials included, and more studies were assessed as high-risk than as low-risk. Nevertheless, low-risk studies tended to report greater benefits and lower heterogeneity. Secondly, there was significant unexplained heterogeneity, and a meta-analysis of subgroups did not help to reduce the heterogeneity. Also, the heterogeneity may be related to the small number of reports. Thirdly, some studies did not describe the manipulations sufficiently enough to allow application in practice, leading to a high risk in the risk-of-bias evaluation. The trials' sizes were different, which indicates that the trial results may not reflect the actual situation and may have resulted in an error. Also, the possibility of publication bias exists, although no statistical evidence for this was found.

\section{Implications for clinical practice}

In this study, we found that acupuncture therapy could relieve pain in acute LBP; however, the conclusion should be interpreted carefully because of the high heterogeneity in our analysis. In future studies, researchers should expand the numbers of included patients to strengthen the conclusions, supply more details about the manipulation of the acupuncture therapy, and standardize trials' performance.

\section{Conclusions}

Our meta-analysis found that acupuncture treatment was associated with modest improvements in the VAS score, ODI score, and NOP, but not with improvement in RMDQ score in patients with acute LBP. Our findings should be considered cautiously because of the methodological weaknesses in the included trials. High-quality trials are needed further to assess the role of acupuncture therapy for acute LBP.

\section{Acknowledgments}

We are appreciative of all the staff that helped to complete this review and would like to express our gratitude to ELSEVIER for writing suggestions.

Funding: This work was supported by the following programs: Guangdong Provincial Bureau of Traditional Chinese Medicine (grant number 20181044). Self-funded Science and Technology Project of Foshan (grant number 2018AB000112). Famous Doctor Support Project of Foshan [grant number (2019) 111 Issued by the Foshan Health Bureau]. Famous Doctor Studio Construction Project of Nanhai District of Foshan [grant number (2019) 65 Issued by Nanhai Health Bureau].

\section{Footnote}

Reporting Checklist: The authors have completed the PRISMA reporting checklist. Available at http://dx.doi. org/10.21037/apm-20-1998

Conflicts of Interest: All authors have completed the ICMJE uniform disclosure form (available at http://dx.doi. org/10.21037/apm-20-1998). The authors have no conflicts of interest to declare.

Ethical Statement: The authors are accountable for all aspects of the work in ensuring that questions related to the accuracy or integrity of any part of the work are appropriately investigated and resolved.

Open Access Statement: This is an Open Access article 
distributed in accordance with the Creative Commons Attribution-NonCommercial-NoDerivs 4.0 International License (CC BY-NC-ND 4.0), which permits the noncommercial replication and distribution of the article with the strict proviso that no changes or edits are made and the original work is properly cited (including links to both the formal publication through the relevant DOI and the license). See: https://creativecommons.org/licenses/by-nc-nd/4.0/.

\section{References}

1. GBD 2015 Disease and Injury Incidence and Prevalence Collaborators. Global, regional, and national incidence, prevalence, and years lived with disability for 310 diseases and injuries, 1990-2015: a systematic analysis for the Global Burden of Disease Study 2015. Lancet 2016;388:1545-602.

2. Vas J, Perea-Milla E, Mendez C, et al. Efficacy and safety of acupuncture for the treatment of non-specific acute low back pain: a randomised controlled multicentre trial protocol (ISRCTN65814467). BMC Complement Altern Med 2006;6:14.

3. Deyo RA, Weinstein JN. Low back pain. N Engl J Med 2001;344:363-70.

4. Carey TS, Garrett JM, Jackman A, et al. Recurrence and care seeking after acute back pain: results of a long-term follow-up study. North Carolina Back Pain Project. Med Care 1999;37:157-64.

5. Friedman BW, Chilstrom M, Bijur PE, et al. Diagnostic testing and treatment of low back pain in United States emergency departments: a national perspective. Spine (Phila Pa 1976) 2010;35:E1406-11.

6. McIntosh G, Hall H. Low back pain (acute). BMJ Clin Evid 2011;2011:1102.

7. Jüni P, Reichenbach S, Egger M. COX 2 inhibitors, traditional NSAIDs, and the heart. BMJ 2005;330:1342-3.

8. Eisenberg DM, Post DE, Davis RB, et al. Addition of choice of complementary therapies to usual care for acute low back pain: a randomized controlled trial. Spine (Phila Pa 1976) 2007;32:151-8.

9. Melchart D, Weidenhammer W, Streng A, et al. Prospective investigation of adverse effects of acupuncture in 97733 patients. Arch Intern Med 2004;164:104-5.

10. Wu MS, Chen KH, Chen IF, et al. The Efficacy of Acupuncture in Post-Operative Pain Management: A Systematic Review and Meta-Analysis. PLoS One 2016;11:e0150367.

11. Lee JH, Choi TY, Lee MS, et al. Acupuncture for acute low back pain: a systematic review. Clin J Pain 2013;29:172-85.

12. Manheimer E, White A, Berman B, et al. Metaanalysis: acupuncture for low back pain. Ann Intern Med 2005;142:651-63.

13. Chou R, Deyo R, Friedly J, et al. Nonpharmacologic Therapies for Low Back Pain: A Systematic Review for an American College of Physicians Clinical Practice Guideline. Ann Intern Med 2017;166:493-505.

14. Savović J, Weeks L, Sterne J, et al. Evaluation of the Cochrane Collaboration's tool for assessing the risk of bias in randomized trials: focus groups, online survey, proposed recommendations and their implementation. Syst Rev 2014;3:37.

15. Hasegawa TM, Baptista AS, de Souza MC, et al. Acupuncture for acute non-specific low back pain: a randomised, controlled, double-blind, placebo trial. Acupunct Med 2014;32:109-15.

16. Kennedy S, Baxter GD, Kerr DP, et al. Acupuncture for acute non-specific low back pain: a pilot randomised nonpenetrating sham controlled trial. Complement Ther Med 2008;16:139-46.

17. Cohen MM, Smit V, Andrianopoulos N, et al. Acupuncture for analgesia in the emergency department: a multicentre, randomised, equivalence and non-inferiority trial. Med J Aust 2017;206:494-9.

18. Shin JS, Ha IH, Lee J, et al. Effects of motion style acupuncture treatment in acute low back pain patients with severe disability: a multicenter, randomized, controlled, comparative effectiveness trial. Pain 2013;154:1030-7.

19. Gu J, Guo Y, Liang Y. Clinical observation of different needle retention times for acute lumbar sprain treated with float needle. Zhongguo Zhen Jiu 2015;35:891-4.

20. Su JT, Zhou QH, Li R, et al. Immediate analgesic effect of wrist-ankle acupuncture for acute lumbago: a randomized controlled trial. Zhongguo Zhen Jiu 2010;30:617-22.

21. Liu LL, Lu J, Ma HF. Clinical trials for treatment of acute lumbar sprain by acupuncture stimulation of "yaotong" and local ashi-points in combination with patients' lumbar movement. Zhen Ci Yan Jiu 2017;42:72-5.

22. Lin W, Liangde $\mathrm{P}$, Yalin S, et al. The effect of electroacupuncture at ashi-point with para-acupuncture method on lumbar mobility in patients with acute lumbar sprain. Hubei Journal of TCM 2016;38:67-69.21.

23. Fei Q, Hongli G, Yanjun L, et al. Clinical observation on treating acute lumbar muscle sprain by electropuncture. Clinical Journal of Chinese Medicine 2017;9:39-40.

24. Lili S, Kai L, Hong S, et al. Clinical Study on Distal- 
points Acupuncture Combined with Exercise for Acute Lumbar Sprain: Experiences from Professor SUN Yuanzheng. Journal of Clinical Acupuncture and Moxibustion 2017,33:38-40.

25. Di W. Clinical effect observation of acupuncture in treating acute lumbar sprain pain. Journal of Clinical Acupuncture and Moxibustion 2013;5:41-2.

26. Guichao P, Shen Y. Clinical observation of beryllium acupuncture in the treatment of acute lumbar sprain due to compression of gluteal epithelium. Chinese Community Doctors 2018;34:82-5.

27. Fei W, Xianglin Z. Efficacy of electro-acupuncture at the Jiaji acupoint and quick impale at the Yaotong acupoint on acute lumbar sprain. Journal of Clinical Acupuncture and Moxibustion 2018;10:41-3.

28. Xiang Y, He JY, Tian HH, et al. Evidence of efficacy of acupuncture in the management of low back pain: a systematic review and meta-analysis of randomised

Cite this article as: $\mathrm{Su} \mathrm{X}$, Qian $\mathrm{H}$, Chen B, Fan W, Xu D, Tang C, Lu L. Acupuncture for acute low back pain: a systematic review and meta-analysis. Ann Palliat Med 2021;10(4):3924-3936. doi: 10.21037/apm-20-1998 placebo- or sham-controlled trials. Acupunct Med 2020;38:15-24.

29. Yuan QL, Guo TM, Liu L, et al. Traditional Chinese medicine for neck pain and low back pain: a systematic review and meta-analysis. PLoS One 2015;10:e0117146.

30. Chiarotto A, Maxwell LJ, Terwee CB, et al. RolandMorris Disability Questionnaire and Oswestry Disability Index: Which Has Better Measurement Properties for Measuring Physical Functioning in Nonspecific Low Back Pain? Systematic Review and Meta-Analysis. Phys Ther 2016;96:1620-37.

31. Smeets R, Köke A, Lin CW, et al. Measures of function in low back pain/disorders: Low Back Pain Rating Scale (LBPRS), Oswestry Disability Index (ODI), Progressive Isoinertial Lifting Evaluation (PILE), Quebec Back Pain Disability Scale (QBPDS), and Roland-Morris Disability Questionnaire (RDQ). Arthritis Care Res (Hoboken) 2011;63 Suppl 11:S158-73. 
Cochrane Central Register of Controlled Trials, Allied and Complementary Medicine (AMED), MEDLINE and EMBASE search strategy via OVID

1. exp Acupuncture Therapy/or exp Acupuncture/or exp Acupuncture Points/

2. exp acupressure/or exp electroacupuncture/or exp meridians/ or exp moxibustion/

3. acupuncture.mp.

4. electroacupuncture.mp.

5. (acupunct\$ or electroacupunct\$ or electro acupunct\$).mp.

6. meridian\$.mp.

7. deqi.mp.

8. moxibustion\$.mp.

9. acupressure.mp. [mp=tx, bt, ti, ab, ct, kw, ot, sh, hw, tn, $\mathrm{dm}, \mathrm{mf}, \mathrm{dv}, \mathrm{fx}, \mathrm{dq}, \mathrm{nm}, \mathrm{kf}, \mathrm{ox}, \mathrm{px}, \mathrm{rx}, \mathrm{ui}, \mathrm{sy}]$

10. (electro next stimulat* or electro next acupuncture).mp. $[\mathrm{mp}=\mathrm{tx}, \mathrm{bt}, \mathrm{ti}, \mathrm{ab}, \mathrm{ct}, \mathrm{kw}, \mathrm{ot}, \mathrm{sh}, \mathrm{hw}, \mathrm{tn}, \mathrm{dm}, \mathrm{mf}, \mathrm{dv}, \mathrm{fx}, \mathrm{dq}$, nm, kf, ox, px, rx, ui, sy]

11. (((electro adj1 stimulat\$) or electro) adj1 acupuncture).tw.

12. randomized controlled trial.pt.

13. controlled clinical trial.pt.

14. randomly.ab.

15. trial.ab.

16. placebo.mp.

17. double blind procedure.tw.

18. Single Blind Procedure/

19. Clinical Article/

20. exp Clinical Study/

21. Clinical Trial/

22. Controlled Study/

23. Major Clinical Study/

24. Multicenter Study/

25. placebo/

26. randomly.ab,ti.

27. (randomized or randomised).ab,ti.

28. dorsalgia.ti,ab.

29. exp Back Pain/

30. backache.ti,ab.

31. (lumbar adj pain).ti,ab.
32. coccyx.ti,ab.

33. coccydynia.ti,ab.

34. lumbago.ti,ab.

35. exp low back pain/

36. (low adj back adj pain).mp.

37. spinal column/

38. back disorder*.mp.

39. sciatica.mp.

40. acute.mp.

41.1 or 2 or 3 or 4 or 5 or 6 or 7 or 8 or 9 or 10 or 11

42.12 or 13 or 14 or 15 or 16 or 17 or 19 or 20 or 21 or 22 or 23 or 24 or 25 or 26 or 27

43. lumbar sprain.mp. [mp=tx, bt, ti, ot, ab, ct, sh, kw, hw, tn, dm, mf, dv, fx, dq, nm, kf, ox, px, rx, an, ui, ds, on, sy] 44. back sprain.mp. [mp=tx, bt, ti, ot, ab, ct, sh, kw, hw, tn, $\mathrm{dm}, \mathrm{mf}, \mathrm{dv}, \mathrm{fx}, \mathrm{dq}, \mathrm{nm}, \mathrm{kf}, \mathrm{ox}, \mathrm{px}, \mathrm{rx}, \mathrm{an}, \mathrm{ui}, \mathrm{ds}, \mathrm{on}, \mathrm{sy}]$

45.28 or 29 or 30 or 31 or 32 or 33 or 34 or 35 or 36 or 37 or 38 or 39 or 43 or 44

46. 40 and 41 and 42 and 45

\section{Search strategy in Chinese databases}

China National Knowledge Infrastructure (CNKI) database (1404) Up to September 5, 2019

$(S U=$ 针尒 ' OR SU =' 针刺 ' OR SU =' 毫针 ' OR SU =' 电针 ' $\mathrm{OR} \mathrm{SU}=$ ' 体针 ' $\mathrm{OR} \mathrm{SU}=$ '头针 ' $) \mathrm{AND}(\mathrm{SU}=$ ' 腰痛 '

$\mathrm{OR} \mathrm{SU}=$ ' 腰扭伤 ' OR SU =' 腰损伤 ') AND (SU =' 急性 ')

Chinese Science and Technology periodical Database (VIP) (1036) Up to September 2019

$(M=$ 针炎 $O R M=$ 针刺 $O R M=$ 毫针 $O R M=$ 电针 $O R M=$ 体针 OR $M=$ 头针 $\mathrm{OR} M=$ 耳针 $\mathrm{OR} M=$ 舌针 $) \operatorname{AND}(M=$ 腰痛 $\mathrm{OR} M=$ 腰扭伤 $\mathrm{OR} M=$ 腰损伤 $) \operatorname{AND}(M=$ 急性 $)$

Wanfang Database (1440)Up to September 2019

(主题: 针尒 OR 主题: 针刺 $\mathrm{OR}$ 主题: 毫针 $\mathrm{OR}$ 主题: 电针 $\mathrm{OR}$ 主题: 体针 $\mathrm{OR}$ 主题: 头针 $\mathrm{OR}$ 主题: 耳针 $\mathrm{OR}$ 主题: 舌针) AND (主题: 腰痛 $\mathrm{OR}$ 主题: 腰扭 伤 OR 主题: 腰损伤) AND (主题: 急性) 
Table S1 Consistency evaluation of risk of bias assessment (kappa coefficient)

\begin{tabular}{ll}
\hline Items & Kappa coefficient \\
\hline Random sequence generation (selection bias) & 1 \\
Allocation concealment (selection bias) & 0.806 \\
Blinding of participants and personnel (performance bias) & 0.806 \\
Blinding of outcome assessment (detection bias) & 0.629 \\
Incomplete outcome data (attrition bias) & 1 \\
Selective reporting (reporting bias) & 1 \\
Other bias & 1 \\
\hline
\end{tabular}

Agreement was judged as poor or fair if kappa coefficient ranging from 0 to 0.4 ; moderate if $0.41 \leq k \leq 0.60$; substantia if $0.61 \leq k \leq 0.80$ and perfect if $0.81 \leq \mathrm{k} \leq 1$. 